\title{
Psoriatic Patient, Methotrexate Is a Good Choice of Drug
}

\author{
Kartick Chanda Shaha ${ }^{* 1}$, Ismat Ara $^{2}$, Syed Golam Yusuf Siddique ${ }^{3}$, \\ Rawshan Umme Salma Jahan ${ }^{4}$, Bhagyoshree Karmokar Jyoti ${ }^{5}$, Manashi Sarker ${ }^{6}$
}

\section{Abstract}

Introduction: Psoriasis is a lifelong autoimmune disease which is characterized by white to red color patches of abnormal skin with patches of itchy and scaly. The term psoriasis derived from Greek word "psora" which means "itch". It is a chronic skin disease, touching about 2\% of worldwide population. Different types of psoriasis have been reported such as plaque psoriasis, psoriatic arthritis, scalp psoriasis, flexural (inverse) psoriasis, guttate psoriasis, pustular psoriasis, nail psoriasis, erythroderma which can be diagnosed by solid conclusion such as skin biopsies etc. Case report: We present the case of a 55-years-old male with psoriasis. The diagnosis of psoriasis is based on the clinical features and history, as well as on histopathological examinations of patient's tissue specimens. Any final diagnosis should include a differential diagnosis which includes severe seborrheic dermatitis, Contact dermatitis, pityriasis rubra pilaris and mycosis fungoides which can be clinically indistinguishable from a severe form of psoriasis. Histopathological studies are also generally needed to achieve a definitive diagnosis. After histopathological confirmation of psoriasis methotrexate therapy has been started. Discussion: After comparing this case with several published articles/case reports on psoriasis we found some similarity regarding presentation, use and good response to methotrexate therapy but dissimilarity regarding response to steroid therapy. Conclusion: Our patient showed an excellent response to methotrexate medication.

Key words: Psoriasis, Scaly lesion, Methotrexate.

Number of Figures: 02; Number of References: 10; Number of Correspondence: 03.

*1. Corresponding Author: Dr. Kartick Chanda Shaha M.B.B.S, M.phil (Pharmacology)

Assistant Professor

Department of Pharmacology

Dhaka National Medical College, Dhaka.

Email: Kartick_shaha@yahoo.com

Mobile Number: 01672971352

\section{Dr. Ismat Ara}

Medical Officer

Department of Dermatology and Venereology SSMCH

3. Dr. Syed Golam Yusuf Siddique

DDV Student

Department of Dermatology and Venereology SSMCH

4. Dr. Rawshan Umme Salma Jahan

DDV Student

Department of Dermatology and Venereology SSMCH

5. Dr. Bhagyoshree Karmokar Jyoti

Lecturer

Department of Biochemistry

Dhaka National Medical College

6. Dr. Manashi Sarker

HMO

Department of Dermatology and Venereology $\mathrm{SSMCH}$

\section{Introduction}

Psoriasis is a chronic inflammatory skin disease with a strong genetic predisposition and autoimmune pathogenic traits. The worldwide prevalence is about $2 \%$, but varies according to regions. It shows a lower prevalence in Asian and some African populations, and up to $11 \%$ in Caucasian and Scandinavian populations ${ }^{1}$. Psoriasis can present at any age and has been reported at birth and in older people of advanced age. Accurate determination of the age of onset of psoriasis is problematic, as studies which do so typically rely on a patient's recall of the onset of lesions or determine the onset from the physician's diagnosis as recorded on the initial visit. The mean age of onset for the first presentation of psoriasis can range from 15 to 20 years of age, with a second peak occurring at 55-60 years ${ }^{2}$. Positive family history for psoriasis patients is common in $30 \%$ up to $90 \%$ of cases, as genetic factors have an important role in the disease susceptibility and expression. Literature findings present a threefold increased risk of developing psoriasis in monozygotic twins compared to fraternal twins ${ }^{3}$. There are different clinical types of psoriasis. The most common of which is chronic plaque psoriasis, affecting $80 \%$ to $90 \%$ of patients with psoriasis. The hallmark of classic plaque psoriasis is well-demarcated, symmetric, and erythematous plaques with overlying silvery scale. Plaques are typically located on the scalp, trunk, buttocks, and extremities but can occur anywhere on the body. Patients might demonstrate nail involvement, which can present without concomitant plaques. Active lesions might be itchy or painful. Psoriasis can also present as an isomorphic response, where new lesions develop on previously normal skin that has sustained trauma or injury. The severity of disease can be helpful in guiding management and is classified as mild, moderate, and severe ${ }^{4}$. Most cases of psoriasis are diagnosed 
clinically, but some pustular forms are difficult to recognize. Punch biopsy of the skin may act as a confirmatory workup procedure for atypical cases and exclude other conditions in cases of diagnostic uncertainty: atopic dermatitis, tinea corporis, pityriasis rubra pilaris, seborrheic dermatitis etc ${ }^{3}$. Psoriasis is a disease that can improve and worsen over time, but it usually does not go away completely. Treating psoriasis can improve the skin and may improve quality of life 5 . Many treatment options exist for psoriasis. The treatment choice depends on the severity of psoriasis and whether you have psoriatic arthritis or other medical conditions. For mild or localized psoriasis, topical treatments, such as topical corticosteroids and topical vitamin D, are commonly used. For moderate to severe psoriasis, 3 categories of treatments are considered-phototherapy, oral medications, and biologic medications. Phototherapy, or light therapy, uses specific ultraviolet light wavelengths to treat psoriasis. Both oral and biologic medications are systemic medications, which means that they affect not only the skin but also other parts of the body. Biologic medications reduce specific inflammation molecules that are abnormally elevated in psoriasis ${ }^{6}$. The Psoriasis Area and Severity Index (PASI) is an assessment tool that doctors and nurses use to record the severity of a patient's psoriasis the health condition defined by inflamed lesions and silver-colored plaques.

\section{Case Report}

A 55 years old male patient was admitted in Sir Salimullah Medical College Hospital on 23/11/19 with the complaints of itching all over the body for 20 days. He also complains of erythematous plaques with silvery scaly lesion all over the body but most prominent in scalp, trunk and extremities. Scales are dry, white, and thick. He noticed bleeding after removal of scale. He does not give any history of hair fall. He was treated with steroid by Quack. After steroid therapy patients condition was deteriorated. For better treatment he was admitted in this hospital. On examination, his temperature was recorded $97^{\circ} \mathrm{F}$, pulse rate 80 beats $/ \mathrm{min}$, and blood pressure $120 / 70$ $\mathrm{mm}$ of hg, Auspitz sign was positive. Then clinically he was diagnosed as a case of psoriasis. During admission time, his PASI score was done and the score was $33.3 \%$. Laboratory investigations such as $\mathrm{CBC}$ with blood film, serum creatinine, liver function test, chest $\mathrm{X}$ ray $\mathrm{P} / \mathrm{A}$ view revealed nothing significant. Skin biopsy was done on admission day. He was treated with cap. Cephradine (500 $\mathrm{mg}$ ), Tab. Fexofenadin (120 mg), Bistar shampoo, Cap. Omeprazole $(20 \mathrm{mg})$ and olive oil during admission time. After three days biopsy report confirmed psoriasis. After confirmation, $10 \mathrm{mg}$ methotrexate was added on every Monday and tab folison was added on every Tuesday for 2 months. After 2 weeks of methotrexate therapy patient's condition was improved. During discharge time his PASI score was $15.3 \%$. Patient was advised to come after 15 days for follow up with the investigation result of Serum creatinine, and liver function test.

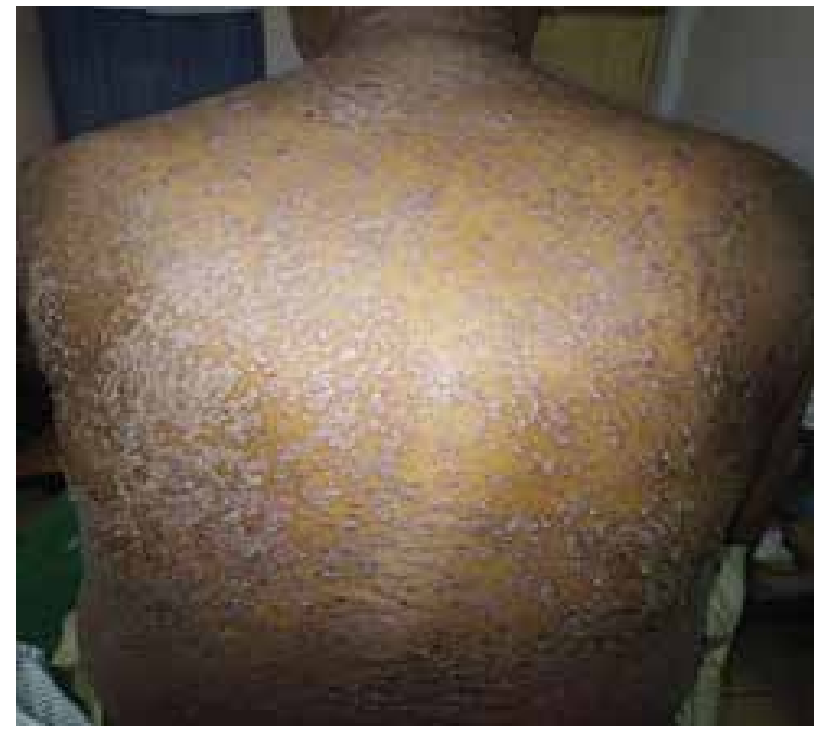

Figure-1: Before methotrexate therapy

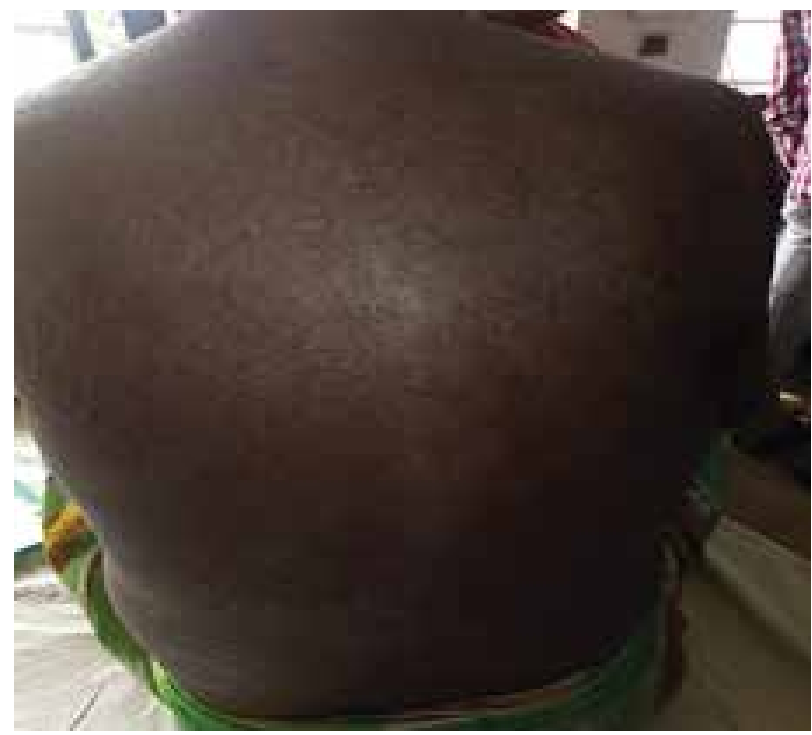

Figure-2: After methotrexate therapy

\section{Discussion}

Our case presented with itching and erythematous plaques with silvery white scale all over the body but most prominent in scalp, trunk and extremities. Dr. R supraja presents a case about psoriatic patient. In his case he reported that his patient present with itching all over the body and patches of $2 \mathrm{cms}$ length covered by scaly lesions which are white in colour and on scrubbing powder like substances falls down and bleeding in drops ${ }^{7}$. The Auspitz sign refers to the appearance of small bleeding points after successive layers of scale have been removed from the surface of psoriatic papules or plaques. In this study the Auspitz sign was present. Bernhard JD conducted a study entitled "Auspitz sign is not sensitive or specific for psoriasis". In his study 41 patients' auspitz sign was positive out of 234 patients with psoriasis ${ }^{8}$. In our study patient's condition was deteriorated after steroid therapy. 
May be the dosage schedule of steroid was wrong because it was prescribed by a Quack. Uva et. al (2012) in his study stated that Corticosteroids remain first-line treatment in the management of all grades of psoriasis, both as monotherapy or as a complement to systemic therapy 9 Our study we found that after methotrexate therapy patient's condition was improved. Martins and lucia (2004) in his study stated that MTX still represents a treatment option with good efficacy/cost/tolerance relationship, especially in poorer countries. The final decision of the therapeutic choice for patient with psoriasis should be based on their needs and preferences, but also on the clinical and economic consequences of the therapeutic strategy adopted, and some studies have confirmed a longer remission time and lower cost in rotating treatments with the use of $\mathrm{MTX}^{10}$.

\section{Conclusion}

Psoriasis is a multisystem inflammatory disease that is underdiagnosed and undertreated despite its prevalence and considerable effect on quality of life. Beyond skin and joint involvement, psoriasis is also associated with an array of important medical and psychiatric comorbidities that require timely therapy to improve long-term outcomes. Our patient showed an excellent response to methotrexate medication.

\section{Conflict of Interest: None.}

\section{Acknowledgement}

I am very much grateful to the dermatology and venereology department of Sir Salimullah Medical College Hospital for their contribution to my study.

\section{References}

1. Adriana rendon and knut schakel. Psoriasis Pathogenesis and Treatment. Int J Moi Sci. 2019; March 20(6): 1475.

https://doi.org/10.3390/ijms20061475

PMid:30909615 PMCid:PMC6471628

2. R G B Langley, G G Krueger, C E M Griffiths. Psoriasis: epidemiology, clinical features, and quality of life. BMJ Journals. vol.64, pp.18-23. https://doi.org/10.1136/ard.2004.033217

\section{PMid:15708928 PMCid:PMC1766861}

3. Orasan, M.S., Roman, I.I. and Coneac, A. Evaluation of psoriatic patients. Published November, 2018.

4. Kim, W.B., Jerome, D. and Yeung, J. Diagnosis and management of psoriasis. Can Fam Physician, vol. April 63 (4), pp. 278-285.

5. Tropy, J.M., Burke, A.E. and Golub, R.M. Psoriasis. JAMA. 2011; 306(8):896.

https://doi.org/10.1001/jama.2011.1162

PMid:21862752

6. Armstrong, A.W. Psoriasis. JAMA Dermatol. 2017; 153(9): 956.

https://doi.org/10.1001/jamadermatol.2017.2103

PMid:28903148

7. Dr. R Supraja. A case report on psoriasis. PMC4147477. 2013 January; vol. 32.

https://doi.org/10.4103/0257-7941.123812

\section{PMCid:PMC4147477}

8. Bernhard JD. Auspitz sign is not sensitive or specific for psoriasis. J Am Acad Dermatol. 1990; 22(6 pt 1): 1079-10781.

https://doi.org/10.1016/0190-9622(90)70155-B

9. Uva, L., Miguel, D., Pinheiro, C., et al. Mechanisms of Action of Topical Corticosteroids in Psoriasis. Int $\mathrm{J}$ Endocrinol. 2012

https://doi.org/10.1155/2012/561018

PMid:23213332 PMCid:PMC3508578

561018. Published online 2012 Nov 5.

10. MARTINS, G. A., Lucia, A. Systemic treatment of psoriasis - Part I: methotrexate and acitretin. An. Bras. Dermatol. May 2004; 79(3): 263-278.

https://doi.org/10.1590/S0365-05962004000300002 